\title{
Web 2.0 vs. the Semantic Web: A Philosophical Assessment
}

\author{
Luciano Floridi ${ }^{1,2}$ \\ ${ }^{1}$ Research Chair in Philosophy of Information and GPI, University of Hertfordshire; ${ }^{2}$ Faculty \\ of Philosophy and IEG, University of Oxford.
}

Address for correspondence: Department of Philosophy, University of Hertfordshire, de Havilland Campus, Hatfield, Hertfordshire AL10 9AB, UK; 1.floridi@herts.ac.uk

\begin{abstract}
The paper develops some of the conclusions, reached in Floridi (2007), concerning the future developments of Information and Communication Technologies (ICTs) and their impact on our lives. The two main theses supported in that article were that, as the information society develops, the threshold between online and offline is becoming increasingly blurred, and that, once there won't be any significant difference, we shall gradually re-conceptualise ourselves not as cyborgs but rather as inforgs, i.e. socially connected, informational organisms. In this paper, I look at the development of the so-called Semantic Web and Web 2.0 from this perspective and try to forecast their future. Regarding the Semantic Web, I argue that it is a clear and well-defined project, which, despite some authoritative views to the contrary, is not a promising reality and will probably fail in the same way AI has failed in the past. Regarding Web 2.0, I argue that, although it is a rather ill-defined project, which lacks a clear explanation of its nature and scope, it does have the potentiality of becoming a success (and indeed it is already, as part of the new phenomenon of Cloud Computing) because it leverages the only semantic engines available so far in nature, us. I conclude by suggesting what other changes might be expected in the future of our digital environment.
\end{abstract}

\section{Keywords}

Cloud Computing; ICT; Inforgs; Infosphere; Semantic Web; Web 2.0. 


\section{Introduction}

What is the next stage in the development of the Web? At least since the dot-com mess, the question has kept pundits and techno-fans on their toes. The recent reshaping of the industry, with the blog-sphere coming to maturity (The Economist, 2008) has only increased the pressure. Recently, two distinct answers have gradually emerged from the rather vociferous and noisy market of ideas: one, unmistakeably Berners-Lee's, advocates the Semantic Web, the other, easily recognisable as O'Reilly's, supports the so-called Web 2.0. As usual, philosophers have been rather quiet on the issue, but it is time to break the silence and take sides. This is what I intend to do in this paper.

In the following pages, I will defend a fairly simple thesis. Semantic Web applications are either exciting science fiction (when "semantic" in Semantic Web is taken seriously) or realistic trivialities (what I shall call the MetaSyntactic Web), whereas it is unclear what Web 2.0 applications really amount to, but they do capture an actual novelty in the current development of online technologies, for they take full advantage of the semantic and collaborative capacities of human users in order to improve and expand the infosphere (http://en.wikipedia.org/wiki/Infosphere). More specifically, in section two, I will argue that the Semantic Web is a clear and well-defined project, which is most definitely not a promising reality, despite some authoritative views to the contrary (e.g., Berners-Lee and Fischetti 1999). I will highlight some of its main problems in section three and argue that the Semantic Web, if taken seriously, will fail in the same way as AI has failed in the past. In section four, I will argue that Web 2.0 (O'Reilly 2006) is a rather ill-defined idea, lacking a clear explanation of its nature and scope. However, I will also argue that current critics, such as Berners-Lee (Anderson 2006), may be compared to detractors of non-AI solutions to problems once interpreted as AI-solvable, such as John McCarthy (1997), who was disappointed by Deep Blue and its ability to win against Kasparov, despite having the intelligence of a toaster. In section five, I will defend the view that, precisely because the Web 2.0 is not the Semantic Web, this is one reason why it is succeeding. For, once the ontological nature of Web 2.0 is made explicit and precise, it can be shown to be a very promising reality, which best captures the future development of current ICTs, since it leverages the only semantic engines available so far in the universe, us, and our social capacities to collaborate cumulatively. In section six, I will comment on how the philosophy of information may help us to understand current technological developments in the information society. I will conclude by briefly commenting on the process of defragmentation of the infosphere in section seven. 


\section{What is the Semantic Web?}

The idea of a Semantic Web was introduced by Tim Berners-Lee in the nineties. A decade or so later, it has become hard to disentangle a simple and clear definition of the Semantic Web, also known as Web 3.0, from a barrage of unrealistic and inflated hype or just unreliable and shameless advertisements. I hope the reader will not mind if I provide a longish selection of quotes. They are necessary in order to illustrate how inflated the idea of a Semantic Web really is, verbatim. The following passages are all from Berners-Lee et al. (2001 all passages retrieved 31 October 2008, emphasis added).

Most of the Web's content today is designed for humans to read, not for computer programs to manipulate meaningfully. Computers can adeptly parse Web pages for layout and routine processinghere a header, there a link to another page - but in general, computers have no reliable way to process the semantics.

The Semantic Web will bring structure to the meaningful content of Web pages, creating an environment where software agents roaming from page to page can readily carry out sophisticated tasks for users. [...] all this without needing artificial intelligence on the scale of 2001's Hal or Star Wars's C-3PO.

[In] The Semantic Web [...] information is given well-defined meaning, [...] as machines become much better able to process and "understand" the data that they merely display at present. [...] To date, the Web has developed most rapidly as a medium of documents for people rather than for data and information that can be processed automatically. The Semantic Web aims to make up for this.

The challenge of the Semantic Web, therefore, is to provide a language that expresses both data and rules for reasoning about the data and that allows rules from any existing knowledge-representation system to be exported onto the Web.

Adding logic to the Web - the means to use rules to make inferences, choose courses of action and answer questions - is the task before the Semantic Web community at the moment. A mixture of mathematical and engineering decisions complicate this task. The logic must be powerful enough to describe complex properties of objects but not so powerful that agents can be tricked by being asked to consider a paradox. Fortunately, a large majority of the information we want to express is along the lines of "a hex-head bolt is a type of machine bolt," which is readily written in existing languages with a little extra vocabulary.

The Semantic Web will enable machines to comprehend semantic documents and data, not human speech and writings. 
Meaning is expressed by RDF [resource description framework], which encodes it in sets of triples, each triple being rather like the subject, verb and object of an elementary sentence. These triples can be written using XML tags. In RDF, a document makes assertions that particular things (people, Web pages or whatever) have properties (such as "is a sister of," "is the author of") with certain values (another person, another Web page). This structure turns out to be a natural way to describe the vast majority of the data processed by machines. Subject and object are each identified by a Universal Resource Identifier (URI), just as used in a link on a Web page.

Human language thrives when using the same term to mean somewhat different things, but automation does not. [...] Using a different URI for each specific concept solves that problem. An address that is a mailing address can be distinguished from one that is a street address, and both can be distinguished from an address that is a speech.

It all makes for fast-paced and exciting reading, full of promises. It is representative of the literature on the Semantic Web. And yet, it is very far from the more cautious and austere perspective endorsed by the World Wide Web Consortium (W3C), which describes the Semantic Web as (emphasis added):

A common framework that allows data to be shared and reused across application, enterprise, and community boundaries. [...] It is based on the Resource Description Framework (RDF)."”

So who is right? And why the notable discrepancy?

\section{Why the Semantic Web won't work}

Supporters of the Semantic Web are, at best, disingenuously naive about its achievability and inadvertedly overenthusiastic about its actual deliverability. A truly semantic web is an AIcomplete problem for which there is no foreseeable, technological solution. ${ }^{2}$ Whereas a technically feasible, allegedly "semantic" Web is unexciting, because it must necessarily fail to deliver what it promises, namely

[...] [a Web in which computers] become capable of analyzing all the data on the Web - the content, links, and transactions between people and computers. A 'Semantic Web', which should make this possible, has yet to emerge, but when it does, the day-to-day mechanisms of trade, bureaucracy and our daily lives will be handled by machines talking to machines. The 'intelligent agents' people have touted for ages will finally materialize." (Berners-Lee and Fischetti, retrieved 31 October 2008).

\footnotetext{
${ }^{1}$ W3C Semantic Web Frequently Asked Questions, http://www.w3.org/RDF/FAQ, retrieved 31 October 2008.

${ }^{2}$ For a very unconvincing view to the contrary see http://semanticwebfaq.com/index.php?title=Is_the_Semantic_Web_artificial_intelligence\%3F
} 
The main problems with the Semantic Web, as envisaged by people like Berners-Lee, may be summarised in the following list.

1) Too much rhetoric and too little detail make the project of a Semantic Web conceptually muddled. Key concepts such as "semantics", “meaning”, "understanding”, “comprehension”, "information", "knowledge" and "intelligence", generously sprinkled on the literature concerning the Semantic Web, are all misused, used too loosely or just metaphorically. The actual facts are that languages, protocols and ontologies for metadata and metasyntax can allow integration, aggregation, sharing, syndication and querying of heterogeneous but wellcircumscribed topic-oriented data, across different databases. Yet there is virtually no "semantics" in this. In 1997, for example, the W3C defined the first Resource Description Framework (RDF) specification, which became a W3C recommendation in 1999. RDF provides triple-based representation language for Universal Resource Identifiers (URIs). No meaning or intelligence plays any role in this.

2) When ambitious, the Semantic Web relies on Strong AI and therefore it is technically unfeasible. But when they try to be more realistic, supporters of the Semantic Web confuse technical feasibility (it can be done in principle) with achievable success (the goals for which the technology is going to be deployed can be reached). One only needs to consider that supersonic civil aviation is still perfectly feasible, yet Concorde was retired in 2003 and there are no serious plans to resurrect supersonic flights. Money may not be an issue (although the current financial downturn does not bode well for large IT projects), yet we should consider very carefully whether we wish to invest in a "Semantic Concorde": some ideas won't fly, no matter how many resources are thrown at them.

3) When modest, the idea of a Semantic Web is much older. As Shadbolt et al. (2006) have remarked "Tim Berners-Lee articulated it at the very first World Wide Web Conference in 1994. This simple idea, however, remains largely unrealized." It still is and, in fact, will remain an old unrealized idea, a direct descendent of Leibniz's dream to design a lingua characteristica (a language in which all knowledge could be formulated unequivocally) and a calculus ratiocinator (calculus of reasoning, basically an inferential semantic engine) such that communication would be vastly improved and disagreements could be resolved more easily. Things have not improved since Leibniz's times, and the whole project of true AI 
remains a dream (Dreyfus, 1992), as the failure to pass even very simplified versions of the Turing test shows (Floridi et al. forthcoming). The world of computer science and ICT has certainly developed, but what we have instead are computers testing users to see whether they are human. The reader will probably have been subjected to (and passed) the test represented by the so-called CAPTCHA ("Completely Automated Public Turing test to tell Computers and Humans Apart"). These are the sort of slightly altered strings of letters that one has to decipher to prove that one is a human not an artificial agent, e.g. when registering for a new account on Wikipedia. Interestingly, a good strategy that computer A can deploy to fool another computer B (say Wikipedia) into believing that A is human is to use a large number of humans as the sort of semantic engines that can solve the CAPTCHA. Computer A connects to computer B, fills out the relevant bits of information (say, an application for a new account on Wikipedia), and then relays the CAPTCHA to a (group of) human operator(s), who are enticed by A to solve it for a reward, without knowing that they are being manipulated (Vaas 2007). This leads me to the following point.

4) Semantic content, in the Semantic Web, is generated by humans, ontologised by humans, and ultimately consumed by humans. Indeed, it is not unusual to hear complaints about how difficult it is to find and retain good "ontologists". RDF, XML, URI and all the other technical solutions are just the mid-stream syntax between a human upstream producer and a human downstream consumer. For example:

The Dublin Core metadata element set is a standard for cross-domain information resource description. It provides a simple and standardised set of conventions for describing things online in ways that make them easier to find. Dublin Core is widely used to describe digital materials such as video, sound, image, text, and composite media like web pages. Implementations of Dublin Core typically make use of XML and are Resource Description Framework based (Wikipedia, "Dublin Core", retrieved 31 October 2008).

It all boils down to dumb taxonomy. No intelligent automatization of semantic processing is envisioned and rightly so.

5) The Return of the AI Zombie. This is a common mistake that seems to be impossible to eradicate once and for all. It consists in confusing the successful climbing of a hill as just the first step towards the moon, instead of the end of the journey. True, ontologies and expert systems have their successful applications in specific contexts, e.g. specific areas in e-science 
or commerce, but it is fanciful to extrapolate from this a success story applicable to the whole web.

6) In connection with the previous point, there is an astonishing underestimation of the difficulties. To quote Shadbolt et al. (2006) again: "The challenges here are real. The ontologies that will furnish the semantics for the Semantic Web must be developed, managed, and endorsed by committed practice communities." But ontologies have a low degree of resilience: tagging, when mistaken, does not cause too much trouble, but an ontology is brittle. Ontologies also suffer from a limited degree of modularity: every bottom-up tag helps immediately, but systematic, top-down, exhaustive and reliable descriptions of entities are useless without a large economy of scale. Tagging a restaurant is already useful per se; providing a restaurant with its URI still makes no difference, unless a lot of other restaurants are equally "URI-ed". If a link fails (the 404 error) one has some positive information about that web page anyway (it is unavailable). If bits of an ontology fail (if a URI fails) the missing information is invisible. What an ontology does not catalogue, the user cannot see. Finally, every ontology depends on a level of abstraction (the choice of a particular interface, to put it simply, see Floridi 2008b) at which the system is conceptualised, yet these levels are neither rigid nor static, but rather flexible and constantly evolving. One may wish to consider a set of restaurants not only in terms of the type of food they offer, but also for their romantic atmosphere, or value for money, or distance from crowded places or foreign languages spoken... the list of potential desiderata is virtually endless, so is the number of levels of abstraction adoptable and no ontology can code every perspective. Finally, metadata are also a brittle solution, and work only partially.

Given the previous clarifications and objections, the truth is that a technically accurate description of a realistically feasible Semantic Web bears very little resemblance to what one finds advertised around. Let me quote the W3C once more (emphasis added):

The Semantic Web is a web of data. [...] The Semantic Web is about two things. It is about common formats for integration and combination of data drawn from diverse sources, where the original Web mainly concentrated on the interchange of documents. It is also about language for recording how the data relates to real world objects. That allows a person, or a machine, to start off in one database, and then move through an unending set of databases which are connected not by wires but by being about the same thing. (http:/www.w3.org/2001/sw/ retrieved 31 October 2008). 
As the reader can see, it is data (not semantic information, which requires some understanding) and syntax (not meaning, which requires some intelligence) all the way through. We should really be speaking of the Machine-readable Web or indeed of the Web of Data as the W3C does. Such MetaSyntactic Web works, and works increasingly well for increasingly circumscribed, standardised and formulaic contexts (e.g., a catalogue of movie DVDs for online customers). This is really what the $\mathrm{W} 3 \mathrm{C}$ is focusing on. Unexciting and, in its true colours, simply unsellable, which is a pity, because the Metasyntactic Web is a genuinely useful development.

\section{What is Web 2.0?}

Nobody has devised a definition of Web 2.0 so far. Providing a watertight list of necessary and sufficient conditions that should qualify something as Web 2.0 might be an impossible rather than just a tricky task. But the fact that Web 2.0 refers to a loose gathering of a wide variety of family-resembling technologies, services and products, is not a justification for a philosophically frustrating lack of clarity. A foggy environment is not a good reason for an out-of-focus picture of it. True, attempts to sharpen what we mean by Web 2.0 applications abound, but none of them has acquired the status of even a de facto standard. So, for our purposes, we might do worse than rely on a sort of self-description. The entry in Wikipedia (which normally qualifies as a Web 2.0 application) states that:

Web 2.0 concepts have led to the development and evolution of web-based communities and its hosted services, such as social-networking sites, video sharing sites, wikis, blogs, and folksonomies. The term became notable after the first O'Reilly Media Web 2.0 conference in 2004. Although the term suggests a new version of the World Wide Web, it does not refer to an update to any technical specifications, but to changes in the ways software developers and end-users utilize the Web. According to Tim O'Reilly: 'Web 2.0 is the business revolution in the computer industry caused by the move to the Internet as platform, and an attempt to understand the rules for success on that new platform." ("Web 2.0", retrieved 31 October 2008).

To be fair, O’Reilly was a bit more precise:

Web 2.0 is the network as platform, spanning all connected devices. Web 2.0 applications are those that make the most of the intrinsic advantages of that platform: delivering software as a continually-updated service that gets better the more people use it, consuming and remixing data from multiple sources, including individual users, while providing their own data and services in a form that allows remixing 
by others, creating network effects through an 'architecture of participation', and going beyond the page metaphor of Web 1.0 to deliver rich user experiences. ${ }^{3}$

So the Semantic Web is really the participatory web, which today includes "classics" such as YouTube, MySpace, eBay, Second Life, Blogger, RapidShare, Facebook and so forth. Just check the top twenty websites in Alexa (www.alexa.com). So what is the difference between Web 2.0 and the Semantic Web? A good way to answer this question is by trying to understand the success of Web 2.0 applications.

\section{Why Web 2.0 works}

Web 2.0 works for the following reasons. Metadata are still data, even if about data, i.e. they are identifiable differences that only afford and constrain (but are still devoid of) semantic interpretation (Floridi 2008a). They should not be confused with semantic information (which requires at least meaning, Floridi 2005), let alone knowledge (which certainly requires truth and may further require at least some form of justification and understanding). However, artificial agents - including everyday software and, as far as current scientific knowledge is concerned, any conceivable software that may be developed in the future - are syntactic engines, which cannot process meaningful data, i.e. information as content, only data at lower- or higher-level. So, the Semantic Web is largely mere hype: we have seen, for example, that XML is a data description language, no information is or can be involved. On the contrary, humans are the only semantic engines available, the ghosts in the machines. So Web 2.0 is the Web created by semantic engines for semantic engines, by relying on the contribution of legions of users. As an illustration, consider folksonomies.

A folksonomy (from folk and taxonomy) is the (aggregated result of) the social practice of producing information (metainformation, to be precise) about other information (e.g., a photograph) through collaborative classification, known as social tagging (e.g., the photograph receives the tags "New York", "Winter", "Statue of Liberty"). It works bottomup, since it is left to the single individual user or producer of the tagged target to choose what to classify, how to classify it and what appropriate keywords to use in the classification. Folksonomies have become popular since 2004, as an efficient way to personalise information and facilitate its fruition through information management tools. Now, it is almost trivial to remark that folksonomies might be egregiously ambiguous, but this is not a

\footnotetext{
${ }^{3}$ Although it seems no longer retrievable from the original website, O'Reilly (2006) can still be googled from many websites.
} 
problem for semantic engines like us, capable of fast disambiguation processes. It turns out that Web 2.0 is an achievable (and increasingly implemented) reality, represented no longer by the creation of another, external space, like Web 1.0, but by an ecosystem friendly to, and inhabited by, humans as inforgs (connected informational organisms).

The previous explanation clarifies that Web 2.0 is part of a space made of information, the infosphere, where memory as registration and timeless preservation (the Platonic view) is replaced by memory as accumulation and refinement. It is an environment characterised by its time-friendliness: time adds value and Web 2.0 applications get better-byuse, that is, they improve with age, not least because the number of people involved is constantly increasing. This, in turn, is a function of critical-mass of "prod-umers", the producers and consumers of semantic information that I have defined above as inforgs. For example: with Wikipedia entries, the longer they are online and used the better ${ }^{4}$ also because a whole new generation of an increasing number of participants escalates the peer-review effect. This explains why Web 2.0 is seen as part of an even more recent development, known as Cloud computing (http://en.wikipedia.org/wiki/Cloud_computing). This is another metaphor (and buzz word) for the Internet, also rather fuzzy and vague. However, as in the case of Web 2.0, Cloud computing does capture a real new paradigm, when it is used to refer to an upgraded utility-fication of computing resources: software tools, memory space, computational power and other services or IT-capabilities are all permanently provided as Internet-based services (in the "cloud") in a way that is entirely infrastructure-transparent and seamless to the user. It is the ultimate challenge to the spatial localization and hence fragmentation of information processes. Cloud computing is space-friendly in the sense that it does not matter where you are but only what computational resources you need.

Web 1.0 and the Semantic Web are, on the contrary, time-unfriendly and fail to rely on the large number of small contributions that can be offered by millions of inforgs. For example, the longer an entry from the Britannica has been available the worse it gets, becoming utterly outdated in the long run; the same applies to old-fashioned web-sites

\footnotetext{
${ }^{4}$ A different (if not contrary view) is expressed by Duguid (2006). This is not the place to provide a detailed analysis of Duguid's intelligent and informed criticism, so I will highlight only two points. First, his objection that Wikipedians should not be so dismissive of the Britannica (or any other published source of information) is correct since, after all, old, copyright free entries from the Britannica are included in Wikipedia, as he remarks. But note that this actually supports the time-friendliness of Wikipedia, since Wikipedia does get better precisely because it can easily cannibalise any other copyright free resource available around. And, second, the editorial structure of Wikipedia is far more complex, articulated and "hierarchical" than people normally seem to acknowledge. Self-generated contents are really the result of hard-driven and highly controlled processes. That anyone can contribute does not mean that anyone may. But this too, is time-friendly, since it relies on volunteers and their willingness to collaborate within an organization.
} 
working as hubs, or any ontology. So, a simple test to know whether something belongs to Web 2.0 is to ask: does it improve with time, usage and hence number of people connected? Services which pass the test are Flickr, YouTube, MySpace, etc.

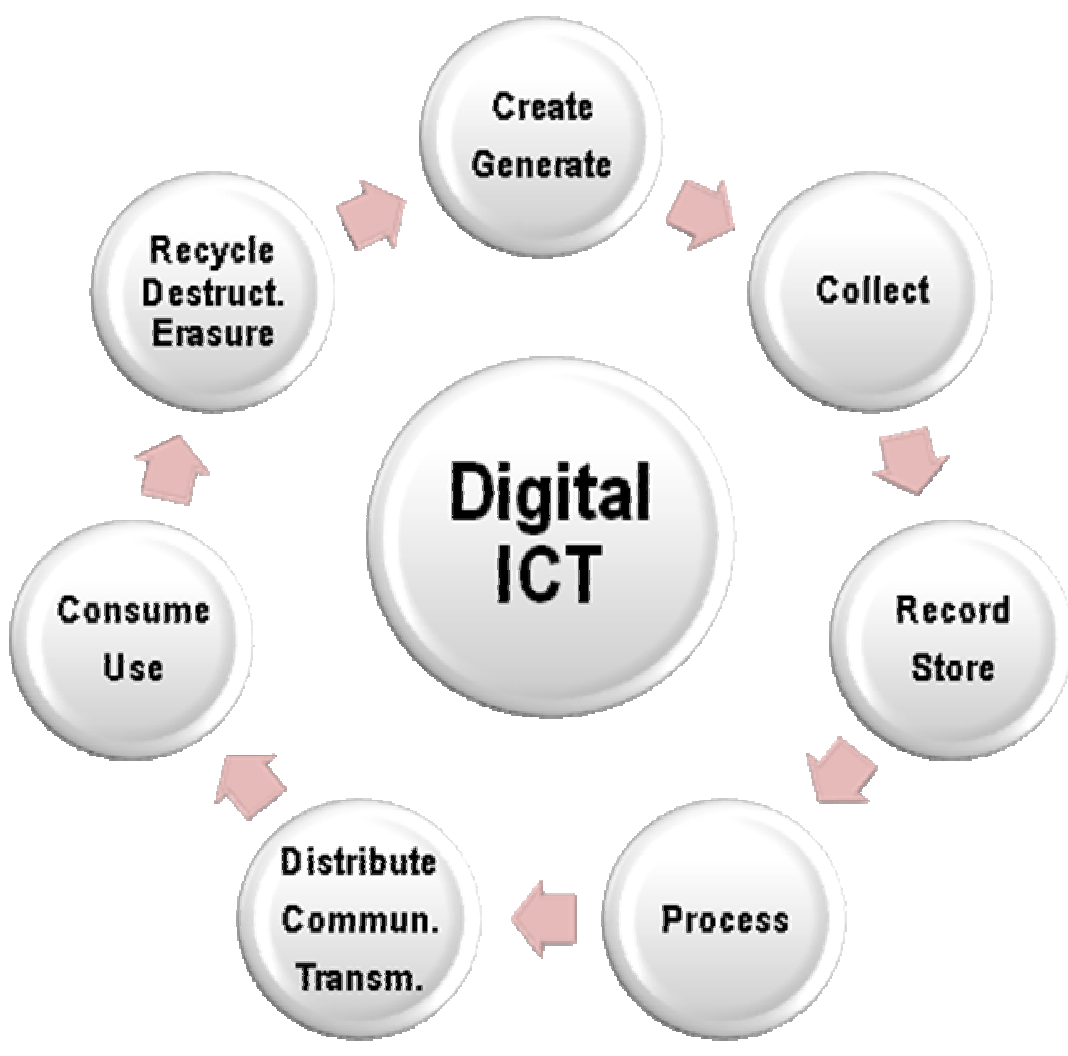

Figure 1. The Life-cycle of Information

\section{The Evolution of the Web: Defragmenting the Infosphere}

To summarise, the full Semantic Web is a well-defined mistake, whereas the Web 2.0 is an ill-defined success. They are both interesting instances of a larger phenomenon, which may be defined as the construction and defragmentation of the infosphere. Web 2.0/the Participatory Web erases barriers between production and consumption of information (less friction) in one or more phases of the information life-cycle (from occurrence through processing and management to usage, see Figure 1), or between producers and consumers of information. Web 3.0/the Semantic Web, understood, as it should, as the MetaSyntactic Web, erases barriers between databases. We might then label Web 4.0 the Bridging Web, which erases the digital divide between who is and who is not a citizen of the information society (effective availability and accessibility). Interestingly, this is happening more in terms of 
smart phones and other hand-held devices - for example in China and India - than in terms of a commodification of personal computers. By Web 5.0 one may then refer to Cloud computing and its ability to erase physical barriers and globalise the local. Finally, Web 6.0 is the Web Onlife, which erases the threshold between here (off-line, analogue, carbon-base) and there (online, digital, silicon-based). In this case, other common labels include "Ubiquitous Computing", "Ambient Intelligence", "The Internet of Things" or "Webaugmented things". These various Webs are developing in parallel and hence are only partially chronological in their order of appearance. Their numbering implies no hierarchical ordering, it is just a matter of convenient labelling. They should be seen more like converging forces pushing the evolution of the web in the direction of a better infosphere.

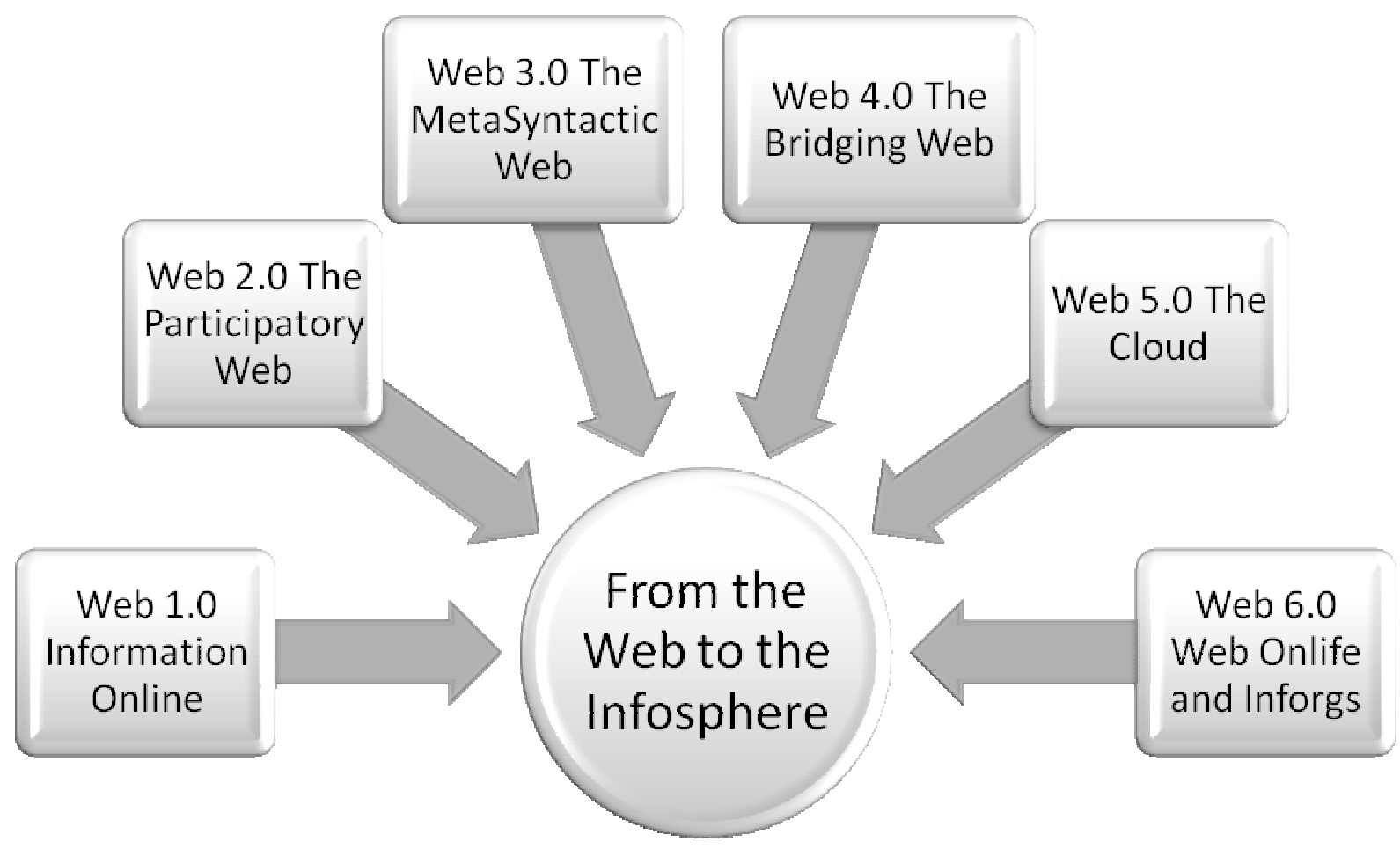

Figure 2: Mapping the Evolution of the Web

\section{Conclusion}

The previous interpretation of the future of the web (see Figure 2) outlines a broad scenario, according to which humans as social inforgs will inhabit an infosphere increasingly boundless, seamless, synchronized (time), delocalised (space) and correlated (interactions). It is an environment based on the gradual accrual and transmission of semantics through time 
by generations of inforgs. A collaborative effort to save and improve meaning for future refinement and reuse: this "green policy" is the last point on which I would like to comment.

The reader may recall the disturbing scenes, in the Matrix, when we are finally shown batteries of humans farmed as mere biological sources of energy. It is a compelling story, but also an idiotic waste of resources. What makes humans special is not their bodies, which are not much better than the bodies many animals have, but that coalition of capacities which one may call intelligence or the mind. We could have tails, horns, wings or plumes, be oviparous or live under the sea: the best use that one could make of humanity as a means would still be in terms of inforgs, organisms that are semantically omnivorous, capable of semantic processing and intelligent interactions. We generate and use meaning a bit like the larvae of the mulberry silkworm produce and use silk. It is an extraordinary feature, possibly unique in the universe, which we have been able to exploit only partially in the past. Civilizations, cultures, sciences, religions, social traditions, languages, narratives, art, music, poetry... in short all the vast semantic input and output of billions of inforgs has been slowly layered for millennia like a thin stratum of humus on the hard bed of history. Too often it has been washed away by natural and man-made disasters, or made sterile by its inaccessibility or unavailability. Without it, human life is the life of a brute, of a mindless body. Yet its presence, preservation, accumulation and best use have been very limited, if compared to what humanity has been able to achieve in the area of management of material and energy resources and shaping of the physical environment. The information revolution (Floridi forthcoming) that we are experiencing today is partly explainable in terms of redressing such a lack of balance. Information and Communication Technologies have reached a stage when they might guarantee the stable presence, the steady accumulation and growth, and the increasing usability of our semantic humus. The good news is that building the infosphere as a friendly environment for future generations is becoming easier. The bad news is that, for the foreseeable future, the responsibility for such a gigantic task will remain totally human. ${ }^{5}$

\footnotetext{
${ }^{5}$ I presented a first version of this paper at Loyola University in July 2007, during NACAP 2007, the yearly North American Computing and Philosophy conference. I am grateful to Selmer Bringsjord, Marvin Croy, Anthony Beavers, Matthew Butcher, George K. Thiruvathukal and Thomas Wren for that opportunity. A revised version was the subject of a talk I gave at University College London in January 2008, and I wish to thank Jon E. Agar and Hasok Chang for their kind invitation. IN both cases, I am very grateful to the participants for the lively discussions I enjoyed during and after the meetings, for they generated several improvements. A special thank goes to Joanna Gillies, for copyediting the text, and to Matteo Turilli, for his useful feedback on a penultimate version of this paper. Finally, I am indebted to Don Fallis for his kind invitation to participate in this special issue, his encouragement and patience with the editorial process, and his many valuable editorial suggestions.
} 


\section{About the author}

Luciano Floridi (www.philosophyofinformation.net) is Professor of Philosophy at the University of Hertfordshire, where he holds the Research Chair in Philosophy of Information, and Fellow of St Cross College, Oxford University, where he is the founder and director of the Information Ethics research Group. He is currently President of the International Association for Philosophy and Computing (www.ia-cap.org) and Gauss Professor of the Academy of Sciences in Göttingen. His forthcoming book is The Philosophy of Information (Oxford University Press). 


\section{References}

Anderson, Nate. 2006. "Tim Berners-Lee on Web 2.0: "Nobody Even Knows What It Means"', Ars Technica. Retrieved 2 November 2008, from http://arstechnica.com/news.ars/post/20060901-7650.html

Berners-Lee, Tim and Fischetti, Mark. 1999. Weaving the Web: Origins and Future of the World Wide Web. San Francisco: HarperCollins.

Berners-Lee, Tim, Hendler, James, and Lassila, Ora. 2001, "The Semantic Web", Scientific American, 284(5), 34-43. Retrieved 2 November 2008 from http://www.sciam.com/article.cfm?id=the-semantic-web.

Dreyfus, Hubert L. 1992. What Computers Still Can't Do: A Critique of Artificial Reason $3^{\text {rd }}$ ed. Cambridge, Mass; London: MIT Press.

Duguid, Paul. 2006. "Limits of Self-Organization: Peer Production and Laws of Quality", $\begin{array}{lllll}\text { First } & \text { Monday. } & \text { Retrieved } & \text { November } & 2008,\end{array}$ http://www.firstmonday.org/issues/issue11_10/duguid/

Floridi, Luciano. 2005. "Information, Semantic Conceptions Of." In Edward N. Zalta (ed.), Stanford Encyclopedia of Philosophy. http://plato.stanford.edu/entries/information-semantic/. Floridi, Luciano. 2007. "A Look into the Future Impact of Ict on Our Lives." The Information Society 23(1): 59-64.

Floridi, Luciano. 2008a. "Data." In William A. Darity (ed.), International Encyclopedia of the Social Sciences. London: Macmillan.

Floridi, Luciano. 2008b. "The Method of Levels of Abstraction." Minds and Machines 18(3): 303-329.

Floridi, Luciano. Forthcoming. "Artificial Intelligence's New Frontier: Artificial Companions and the Fourth Revolution." Metaphilosophy.

Floridi, Luciano, Taddeo, Mariarosaria, and Turilli, Matteo. Forthcoming. "Turing's Imitation Game: Still a Challenge for Any Machine and Some Judges." Minds and Machines. McCarthy, John. 1997. "AI as Sport." Science 276(5318): 1518-1519.

O'Reilly, Tim. 2006. "Web 2.0 Compact Definition: Trying Again". Retrieved March 4, 2007, from http://radar.oreilly.com/archives/2006/12/web_20_compact.html.

Shadbolt, Nigel, Berners-Lee, Tim, and Hall, Wendy. 2006. "The Semantic Web Revisited." IEEE Intelligent Systems 21(3): 96-101.

The Economist. November the $8^{\text {th }} 2008$. "Blogging - Oh Grow UP". 
Vaas Lisa. 2007. “Striptease Used to Recruit Help in Cracking Sites”. Retrieved 31 October, 2008, from http://www.pcmag.com/article2/0,2817,2210674,00.asp 